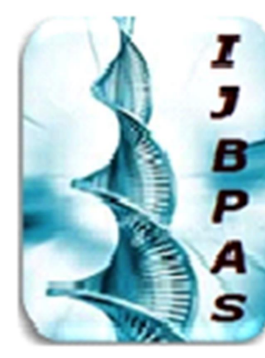

International Journal of Biology, Pharmacy and Allied Seiences (IJBPAS) 'A Bridge Betusen Caboratory and QRendo'

WwW.ijbpas.com

\title{
EFFECT OF YONIVARTI WITH ABHYANTARA CHIKITSA IN KAPHAJ YONIVYAPAD -A CASE STUDY
}

\section{PATEL SP ${ }^{1}$ AND KARKARE $M^{2 *}$}

1: PG Scholar ${ }^{1}$, PTSR Department, Parul Institute of Ayurveda, Parul University, Limda, Vadodara, Gujarat

2: Professor \& HOD of PTSR Department, Parul Institute of Ayurveda, Parul University, Limda, Vadodara, Gujarat

3: Final year PG Scholar, Department of Prasuti Tantra Evum Stree Roga, Parul Institute Of Ayurveda, Vadodara, Gujarat

*Corresponding Author: Dr. Karkare M; E Mail: mrkarkare@gmail.com Received 12 ${ }^{\text {th }}$ Dec. 2021; Revised 14 $4^{\text {th }}$ Jan. 2022; Accepted $7^{\text {th }}$ Feb. 2022; Available online $5^{\text {th }}$ March. 2022 https://doi.org/10.31032/IJBPAS/2022/11.3.1026

\begin{abstract}
Health status of women is essential for healthy society. In today's fast and competitive word, there is change in lifestyle of women which lead to their physical \& mental stress. Women mostly get affected by this environment and are prone to gynaecological problem. Common health problem in women is white discharge, Foul smelling discharge, itching vulvar region, this all are symptoms of kaphaj yonivyapad $.40 \%$ of women suffer from this problem but neglect the problem. Dosha involved in kaphaj yonivyapad is Kaphapradhan vata. Panchkashaya yonivarti with Jeerakadi vati is used to terminate the samprapti of this disease. Yonivarti is a modality of treatment which acts at the site of the disease. This case study is an attempt to evaluate the practicle application of yonivarti with Aabhyantar chikitsa in management of kaphaja yonivyapad.
\end{abstract}

Keywords : Yonivarti, Kaphaj yonivyapad,shaman chikitsa, Sthanik chikitsa 


\section{INTRODUCTION :}

Ayurveda is rich in pharmaceutical preprations but only few preprations are being used in today's Ayurvedic practice because of inconvenient forms. In the management of kaphaj yonivyapad many kalpana like yoniprakshalan, yonipichu, yonivarti are mentioned. Nearly $40 \%$ of women in reproductive age group suffer from white discharge. Modern line of treatment includes antibiotics, antifungal, antihistaminics, which gives temporary relief. Most common cause of symptomatic vaginal discharge is bacterial vaginosis (33$47 \%$ ), followed by candidiasis (20-40\%) and trichomoniasis $(8-10 \%)$. These three organisms account for $90 \%$ of all the of abnormal vaginal discharges. This study was planned to evaluate the efficacy of panchkashaya yonivarti with jeerakadi vati in kaphaja yonivyapad.

2. AIM:

- To evaluate the efficacy of panchkashaya yonivarti with jeerakadi vati in kaphaja yonivyapad.

\section{OBJECTIVE :}

- To study and understand the etiopathogenesis of kaphaja yonivyapad.
- To study the clinical effect of shaman chikitsa with yonivarti in kaphaja yonivyapad.

\section{Case report :}

Study center : Parul Ayurveda Hospital ,Vadodara Gujarat

Name of patient : XXX

Reg OPD No : 21017383

Reg IPD No : :212402

Date of $1^{\text {st }}$ visit $: 20 / 8 / 21$

Region : Hindu

Occupation : Housewife

\section{Chief complain:}

- Yonigata shweta srava since 2-3 years.

- Yonikandu since last 3 years.

- Yonidaurgandhya since last 1 year.

\section{Associated Complaints:}

- Katishula since 3 years.

\section{HISTORY OF PRESENT ILLNESS:}

A 48 years female patient suffering yonigata shweta srava since last 2-3 years was visited the striroga-prasutitantra OPD of Parul ayurveda hospital (PAH). She was suffering from yonigata shweta srava(white discharge $\mathrm{p} / \mathrm{v}$ ) which is mucoid in nature, katishula (Lowerbackache), yonikandu (itching at vulva) since last 3 years. A detailed history of the patient was taken and all required physical and clinical examinations were 
done. She had taken treatment earlier privately but didn't get satisfactory relief in complaints.

\section{Past treatment history:}

- Vaginal tablets and cream

- Oral antibiotics

History of past illness: No any major illness. History of surgery : No history of surgery

Family history : No any Family history

\section{Menstrual history:}

- Age of Menarche : 13 years

- LMP : $2 / 8 / 21$

- Menstrual cycle :

$$
\begin{array}{ll}
\text { Regularity } & : \text { Regular } \\
\text { Duration } & : 2-3 \text { days } \\
\text { Interval } & : 28-30 \text { days } \\
\text { Association of pain }: \text { no } \\
\text { Quantity } \quad: 2-3 \text { pads/day }
\end{array}
$$

(Moderate flow)

Not associated with clots and foul smell

- Marital History :

Married since 23 years.

No history of any type of contraception .

- Obstetics History :

P4L4D0A0

P1:P2:P3:P4:All FTND at Hospital

Personal history:
Diet : Mixed-veg / nonveg

Dietetic habits : Samasana

Rasa Sevana : Madhura, Tikta

Appetite : Poor

Bowel : Regular

Micturation : 4-5 times /day ; 1-2 times /day

Kostha : Madhyam

Thirst : Normal

Personal Hygiene : Poor

Sleep : Disturbed

Sexual Life : Satisfactory

\section{General Examination:}

Height $\quad: 158 \mathrm{~cm}$

Weight $: 65 \mathrm{~kg}$

BMI $\quad: 26 \mathrm{~kg} / \mathrm{m} 2$

Pulse $\quad: 80 / \mathrm{min}$

B.P. $\quad 120 / 80 \mathrm{~mm}$ of $\mathrm{Hg}$

R.R. $\quad: 18 / \min$

Temp : $98.6 \mathrm{~F}$

Tongue : Slightly coated

Pallor/cyanosis /Oedema : Absent

\section{Systemic Examination:}

RS, CVS, CNS, and GIT- NAD

\section{Astavidha pariksha :}

- Nadi $=86 / \mathrm{min}$, madhyama bala

- Mala = Grathita mala pravrutti

- Mutra =Samyak

- Jihva =Saam

- Shabda $=$ Avishesh 
- Sparsh =Anushna

- Druka $=$ Panduvarna

- Akruti $=$ Sthul

Per abdomen Examination:

- Abdomen was soft, No organomegaly

- No tenderness present.

Per speculam: white discharge +++ , cervix Inflammed

Per vaginal examination: ut RV/Mobile

fornicis nontender

Investigation (23/8/2021)

- CBC : wbc:6900/cumm

$\mathrm{Hb}: 9.0 \mathrm{~g} / \mathrm{dl}$

Rest : within normal limit

ESR: $45 \mathrm{~mm}$

- Urine routine micro : Pale yellow in colour, clear, P.H.:6.0 Sp G :
1.015,Protein:Nil, Blood : Nil ,pus cell ;2-3 /HPF

- Vaginal swab culture (21/8/2021): the smear shows moderate puss cell \& no organism.

\section{Samprapti ghatak:}

Dosha : kaphapradhan vata

Dushya : rasa ,mansa

Srotas : rasavaha, aartavavaha

Srotodushtiprakara: Atipravrutti, vimarg gaman

Marga : abhyantara

Agni : Mandagni

Aam : Sama

Udbhava sthana :pakvashaya

Vyaktasthana: Yoni

Samprapti:

The prakruti of patient being vata -Kaphapradhan

ת)

She was predisposed to Kapha-vataprakopa Hetusevan like

\&

Aaharajanya nidana- Ruksha Annapan (Dry foods), Aniyamita Ahara sevana (Irregular food habits), Dadhi sevan

Viharajanya Nidana- Diwaswap (Day sleep), Poor vaginal Hygine<smiles>[CH]</smiles>

All this Nidans lead to Kha-vaigunya in trayavarta yoni<smiles>[CH]</smiles>

Yoni Dushti in the form of inflammation presenting as mucoid white discharge per vagina, ithching per vagina, Foul smelling Discharge and Backche. 


\section{Intervention:}

\section{Sthanika chikitsa:}

- Yoni prakshalana with luke warm water.

- Yonivarti

\section{Method of yonivarti:}

Poorvakarma :

- Counselling was done.

- Written consent was taken.

- Shaving and part prepration .

- Patient was advised to empty the bladder.

- Instrument trolley was prepared.

- Yoni prakshalana with luke warm water done.

\section{Pradhana karma:}

- Private part was cleaned antiseptically.

- Sim's speculum was inserted; Anterior vaginal wall retractor was introduced to expose the cervix.

- Prepared varti was introduced to endocervix.

- Instruments was removed out.

\section{Paschat karma:}

- Patient was monitored for 30 minutes after the procedure.

\section{Safety precautions:}

- Instruction was given to patient to avoid sexual intercourse, heavy work, journey, etc,. during and for 7days after the procedure.

\begin{tabular}{|c|c|c|}
\hline & Sthanika Chikitsa & Abhyantara Chikitsa \\
\hline Drug & $\begin{array}{c}\text { Panchkashaya yoni varti } \\
\text { (vacha,vasa, patol, priyangu,nimba) } \\
\text { (for external use) }\end{array}$ & $\begin{array}{c}\text { Jeerakadi vati } \\
\text { Jeerak, Krishna-jirak, } \\
\text { Pippali,Karvellak,Vacha,Vasa, } \\
\text { Saindhava, Yavakshara,Yavanika }\end{array}$ \\
\hline Dose & $\mathbf{3}$ gm & 500mg 1 vati BD A/F \\
\hline Duration & 7 Days & 7 Days \\
\hline Follow up & $\mathbf{8}^{\text {th }}$ day & \\
\hline
\end{tabular}

Pathya-apatya: She was advised to follow the follow Ahara-Vihara Pathyas as follows

Ahara 1. Drink plenty of water.

2. Eat fibrous diet, fruits, green vegetables.

3. Garlic, meat soup, rice water

Vihara 1. Keep the area clean and dry.

2. Maintain personal hygiene.
3. Wash the garments in boil water and Dettol and then dry it in sunlight. 


\begin{tabular}{|c|c|c|c|}
\hline Day & Date & Treatment given & Observation \\
\hline 1 & $20 / 08 / 2021$ & $\begin{array}{l}\text { Panchakashaya yonivarti } \mathrm{F} / \mathrm{b} \\
\text { sukhoshna jalaprakshalan } \\
\text { 2. Jeerakadi vati 500mg } 1 \text { vati BF } \\
\text { BD }\end{array}$ & $\begin{array}{ll}\text { - } & \text { Mucoid white discharge } \mathbf{P} / \mathbf{v} \\
& +++ \\
\text { - } & \text { Itching p/v +++ } \\
\text { - } & \text { Foul smellind discharge }+++ \\
\text { - } & \text { Backache }+++ \\
\end{array}$ \\
\hline 3 & $22 / 082021$ & Same as above & $\begin{array}{ll}\text { - } & \text { Mucoid white discharge } \mathbf{P} / \mathbf{v} \\
+++ & \\
\text { - } & \text { Itching } \mathbf{p} / \mathbf{v}++ \\
\text { - } & \text { Foul smellind discharge }++ \\
\text { - } & \text { Backache }+++ \\
\end{array}$ \\
\hline 5 & $24 / 08 / 2021$ & Same as above & $\begin{array}{ll}- & \text { Mucoid white discharge } \mathbf{P} / \mathbf{v} \\
++ & \\
\text { - } & \text { Itching } \mathbf{p} / \mathbf{v}+ \\
\text { - } & \text { Foul smellind discharge }+ \\
\text { - } & \text { Backache }++\end{array}$ \\
\hline 7 & $26 / 08 / 2021$ & Same as above & $\begin{array}{ll}- & \text { No Mucoid white discharge } \\
& \text { P/v } \\
\text { - } & \text { No Itching p/v } \\
\text { - } & \text { No Foul smellind discharge } \\
\text { - } & \text { Backache + }\end{array}$ \\
\hline
\end{tabular}

On last day her all symptoms were reduce like white discharge $\mathrm{p} / \mathrm{v}$, Itching $\mathrm{p} / \mathrm{v}$, Foul smelling discharge, Backache. Panchkashaya yonivarti use in therapy has cleaned washed out the secretions and discharge of vagina. Varti helps medicine to remain for longer period for better action it diminishes the chance of infection in genital tract.

\section{DISCUSSION}

The kaphaja yonivyapad is mainly due to vata -kaphakar hetusevan .This disease can be cured mainly by observing hygiene. Personal Hygiene is very important for every woman. In this patient the kaphaja yonivyapad deals with the vitiation of vata and kapha dosha, Pancha kashaya yonivarti and Jeerakadi Vati having specially vatakaphahar properties by which it breaks the samprapti.

\title{
Panchkashaya yonivarti:
}

\author{
दुर्गधा पिच्छिला वाड़पि चूरणे पंचककषायजे : । \\ पंचककषायज := वचा वासा पटोल प्रियंगु निम्ब : । \\ (bhav.ma.kha. chi.70/46)
}




\begin{tabular}{|c|c|c|c|c|c|c|c|c|}
\hline Name & B.N. & Family & Part use & Rasa & Guna & veerya & Vipak & Karma \\
\hline $\begin{array}{l}\text { 1.vacha } \\
\text { (1 part) }\end{array}$ & Acorus calamus & Acoraceae & Rhizome & $\begin{array}{c}\text { Katu,tikta, } \\
\text { kashay (anu) }\end{array}$ & $\begin{array}{c}\text { Laghu, } \\
\text { ushna }\end{array}$ & ushna & katu & $\begin{array}{l}\text { Kapha } \\
\text { vathar }\end{array}$ \\
\hline $\begin{array}{c}2 . v a s a \\
(1 \text { part })\end{array}$ & $\begin{array}{c}\text { Adhatoda } \\
\text { vasika }\end{array}$ & Acantheceae & leafe & $\begin{array}{c}\text { Tikta, } \\
\text { kashaya }\end{array}$ & $\begin{array}{l}\text { Laghu } \\
\text {,ruksha }\end{array}$ & sheeta & katu & $\begin{array}{c}\text { Kapha pitta } \\
\text { har }\end{array}$ \\
\hline $\begin{array}{l}\text { 3.patola } \\
\text { (1 part) }\end{array}$ & $\begin{array}{c}\text { Trichosanthesdi } \\
\text { oica }\end{array}$ & Cucurbitaceae & fruit & $\begin{array}{c}\text { Tikta, } \\
\text { Katu }\end{array}$ & $\begin{array}{c}\text { Laghu,ruk } \\
\text { sha }\end{array}$ & sheeta & Katu & $\begin{array}{c}\text { Kapha } \\
\text { pittaghna }\end{array}$ \\
\hline $\begin{array}{l}\text { 4.priyangu } \\
\text { (1 part) }\end{array}$ & $\begin{array}{c}\text { Calicarpa } \\
\text { marcophylla }\end{array}$ & Verbenaceae & root & $\begin{array}{c}\text { Tikta,kashaya, } \\
\text { madhur }\end{array}$ & $\begin{array}{l}\text { Laghu, } \\
\text { ruksha }\end{array}$ & $\begin{array}{c}\text { Sheeta, } \\
\text { katu }\end{array}$ & katu & $\begin{array}{l}\text { Tridosha } \\
\text { shamak }\end{array}$ \\
\hline $\begin{array}{l}\text { 5.nimba } \\
\text { (1 part) }\end{array}$ & $\begin{array}{l}\text { Azacardia } \\
\text { indica }\end{array}$ & Maliaceae & leafe & Tikta, kashay & $\begin{array}{l}\text { Laghu } \\
\text {,ruksha }\end{array}$ & sheeta & katu & Pittahara \\
\hline
\end{tabular}

- Vacha : Vatahara, Kaphahara, Krimihara

- antibacterial activity of acoruscalamus against Escherichia coli, Staphylococcus aureus, and Candida albicans have been studied. (11) Antibacterial effect of Acorus calamus extractions against gram positive and negative bacteria have been studied (12) antifungal activity demonstrated by Pratibha raval. (13)

- Vasa : The plant contains alkaloids such as Vasicine, vasicinone, deoxyvasicine, vasicol, adhatodinine, and vasicinol Other constituents include vitamin $\mathrm{C}$, saponins, flavonoids as well as steroids, and fatty acids .Vasicine is reported to have uterine stimulant effects . Vasicine acetate showed antimycobacterial activity; the high phenolic content of essential oils contributes to their antimicrobial properties. (14), (15)

- Patola : Vranasodhana, Vrana ropana

- Antifungal activity Hariti and Rathee have stated that the fixed oil of seeds of Trichosanthes species including $\mathrm{T}$. dioica has antifungal property. [16] Antibacterial activity Hariti and Rathee have showed antibacterial activity of the unsaponifiable fraction of the fixed oil of $T$. dioica seeds against Bacilus anthracis and Xanthomonas malracearum. [17] T.dioica has been reported to have Chemo Preventive Property. The Chemo Preventive Property of T.dioica was studied by Sanjib S Bhattacharya, Pallab Kanti P K Haldar.(18) Bhattacharya S,Haldar P K.. studied Protective role of triterpenoid enriched extract of Tdioica root against experimentally 
induced pain and inflammation in rodents.(18) Shivhare et al. reported the wound healing potential of methanolic $(\mathrm{MeOH})$ extract of $T$. dioica (19)

- Priyangu : Daurgandhyahara, Vrana ropan

- callicarpa macrophylla contains diterpene, diterpenoid,3b, 16a,17trihydroxyphyllocladane,flavonoids.having antimicrobial and anti inflammatory property.

- Nimba : Vrana, Kushta, Krimiroga, Visaroga

- The most important active constituent is azadirachtin and the others are nimbolinin, nimbin, nimbidin, nimbidol, nimbanene, nimbandiol, nimbolide, nimbiol, sodium nimbinate, 6- desacetylnimbinene, gedunin, salannin, quercetin, ascorbic acid, amino acids, n-hexacosanol, $\beta$ sitosterol, 7-desacetyl-7benzoylazadiradione, 7- desacetyl-7benzoylgedunin, $17-$ hydroxyazadiradione, polyphenolic flavonoids. Neem constituents having antibacterial, antifungal, antimalarial, antiviral, antihyperglycemic, antiulcer, antioxidant, anticarcinogenic and other biological activities.

\section{Jeerakadi vati:}

जीरक द्वितय कृष्णा सुषवी सुरभिर्वचा ।

वासक: सैन्धवश्च अपि यवक्षार यवानिका । ।

एषा चूर्ण घृते किंचिद भृष्टवा खण्डेन मोदकम ।

कृत्वा खादेध्य अथवा वह्नि योनिरोगादी विमुच्यती। ।

(भ/ प म. योनिरोग 70/49) 


\begin{tabular}{|c|c|c|c|c|c|c|c|c|}
\hline Name & B.N. & Family & Part use & rasa & Guna & Veerya & vipak & Karma \\
\hline $\begin{array}{l}\text { 1.jeerak } \\
\text { (1 part) }\end{array}$ & $\begin{array}{l}\text { Cuminum } \\
\text { cyminum }\end{array}$ & Umbelliferae & Seed & katu & $\begin{array}{l}\text { Laghu } \\
\text {,ruksha }\end{array}$ & Ushna & Katu & $\begin{array}{l}\text { Kapha } \\
\text { vathar } \\
\text {,pittakar }\end{array}$ \\
\hline $\begin{array}{l}2 \text {. Krishna } \\
\text { jeerak } \\
(1 \text { part })\end{array}$ & Carum carvi & Umbelliferae & Seed & katu & Ruksha & Ushna & Katu & $\begin{array}{l}\text { Kapha } \\
\text { har }\end{array}$ \\
\hline $\begin{array}{l}\text { 3.pippali } \\
\text { (1 part ) }\end{array}$ & Piper longum & Piperaceae & Fruit & katu & $\begin{array}{l}\text { Laghu, } \\
\text { Tikshna }\end{array}$ & Ushna & Madhur & $\begin{array}{l}\text { Vat } \\
\text { kapha } \\
\text { har }\end{array}$ \\
\hline $\begin{array}{l}\text { 4.karvellak } \\
\text { (1 part ) }\end{array}$ & $\begin{array}{l}\text { Momordia } \\
\text { churantia }\end{array}$ & Cucurbitaceae & $\begin{array}{l}\text { Pancha } \\
\text { ng }\end{array}$ & Tikta, katu & $\begin{array}{l}\text { Laghu } \\
\text {,ruksha }\end{array}$ & Ushna & Katu & Pittagna \\
\hline $\begin{array}{l}\text { 5.vacha } \\
\text { (1 part ) }\end{array}$ & $\begin{array}{l}\text { Arocus } \\
\text { calamus }\end{array}$ & Acoraceae & rhizome & Katu, tikta & $\begin{array}{l}\text { Laghu } \\
\text {,tikshna }\end{array}$ & Ushna & Katu & $\begin{array}{l}\text { Kapha } \\
\text { vatghna }\end{array}$ \\
\hline $\begin{array}{l}\text { 6.vasa } \\
\text { (1 part ) }\end{array}$ & $\begin{array}{l}\text { Adhatoda } \\
\text { vasika }\end{array}$ & acantheceae & Leafe & $\begin{array}{l}\text { Tikta } \\
\text { kashaya }\end{array}$ & $\begin{array}{l}\text { Laghu, } \\
\text { ruksha }\end{array}$ & Sheeta & Katu & $\begin{array}{l}\text { Kapha } \\
\text { pitta har }\end{array}$ \\
\hline $\begin{array}{l}\text { 7.yavani } \\
\text { (1 part ) }\end{array}$ & $\begin{array}{l}\text { Carum } \\
\text { roxburghinu } \\
\text { m }\end{array}$ & Umbelliferae & Seed & $\begin{array}{l}\text { Katu, } \\
\text { tikta }\end{array}$ & $\begin{array}{l}\text { Laghu, } \\
\text { ruksha, } \\
\text { tikshna }\end{array}$ & Ushna & Katu & $\begin{array}{l}\text { Kapha } \\
\text { vathar }\end{array}$ \\
\hline
\end{tabular}

1. Jeeraka :

- Vatakapha saman, DeepanPachana, Vatanuloman,

Shulaprashaman, Krumighna, Garbhashaya-vishodhana.

- Cuminum cyminum contained: alkaloid, coumarin, anthraquinone, flavonoid, glycoside, protein, resin, saponin, tannin and steroid. Cuminum cyminum having antimicrobial, anti-inflammatory, analgesic, antioxidant, anticancer, antidiabetic, antiplatelet aggregation, hypotensive, bronchodilatory, immunological, contraceptive, anti-amyloidogenic effects(21)

2. Krishna jeeraka :
- Daurgandhyahara, Rochana, Deepan, Pachana, Garbhashaya sodhana

- carum carvi contain mainly carvacrol, carvone, $\alpha$-pinene, limonene, $\gamma$-terpinene, linalool, carvenone, and $p$-cymene.

- The antibacterial activity of carvacrol (5-isopropyl-2methylphenol) is amply documented in various experimental studies (22)

- Monoterpenes like anethofuran, carvone, and limonene occurring in cumin and caraway oil have specifically been highlighted for anticarcinogenic action. (23) 
- An aqueous and an ethanolic extract of caraway seeds produced significant antifertility effect via modulation of follicle stimulating hormone (FSH) and leutinizing hormone (LH) levels, while the estrogen levels were increased. (24)

3. Vatahara,Kpahahara,Rasayan,Krimi, Kustha, Sula

- fruits contain the alkaloid piperine,

- various studies proven the antiinflamatory, antiamoebic, antibacterial activity (25)(26)(27)

4. Karvellak: Vatahara, Kaphahara, Bhedi, Kustha, krimiroga

- The fruits and leaves contain alkaloids, glycoside, saponin-like substances, resin, an aromatic volatile oil and mucilage. Reports also show that the plant has antitumor and anti-HIV activities. (28)

5.Vacha

Vatahara,Kaphahara,Krimihara

6.Vasa : Kaphapittahara,

7. Saindhava :Tridoshanashak, Avidahi

- Agnideepana, Pachana, Ruchya, Netriya, Hridya, Vrishya

8.Yavakshara :Tridoshanashak, Avidahi

9.Yavani :Rochana, Deepan,

Pachana, Vatanuloman, Krimighna
- Compounds found in Carum roxburghianum were $\alpha$-pinene $(0.42 \%), \beta$-pinene $(0.15 \%)$, myrcene $(0.22 \%), \Delta$-carene $(3.27 \%)$, limonene $(16.43 \%), \quad \alpha$-terpinene $\quad(1.44 \%)$, thymol (3.61\%), linalool (1.07\%), carvacrol $(14.68 \%)$, carvone $(2.29 \%)$, 1 -cadinene $\quad(0.14 \%), \quad \beta$-elemene (0.99\%), $\alpha$-humulene (1.27\%), elemol $(38.56 \%)$ and cadinol (14.42\%). The percentage of unidentified components was $(0.93 \%)$.

- C. roxburghianum was very active against bacteria and yeasts(29)

- The antimicrobial activity of essential oil of Carum roxburghianum were carried out and the oil showed antimicrobial activity against two gram positive, two gram negative bacteria and four fungi.(30)

- Jeerakadi modaka mentioned by Bhavaprakasha contains Swetajeerak, Krishnajeerak, Pippali, Karvellak, Vacha, Vasa, Saindhavalavana, Yavakshara, Yavanika.All the ingredient having mainly katu, tikta rasa ,laghu,ruksha guna and ushna veerya .It has vedanaasthapana, vatahara, anulomana and rasayana properties. 
- The drugs vacha ,vasa, patola, priyangu, nimba has been used in yonivarti .

all the dravya having tikta Kashaya rasa ,laghu ruksha guna and katu vipak due to its ruksha guna it is Kapha samak .By going through each ingredient's rasa, guna, virya, vipak, prabhava and karma, we came to know that each and every ingredient has the property of vata and kapha saman individually. Insertion of yonivarti is a practical procedure which is performed by the disinfectant and medicated yonivarti . Yonivarti are meant for insertion into vagina, for the purpose of removal of accumulated dosha at local site, for reducing vaginal discharge, Foul smell and Pain. It is one of the upakrama of sthanika chikitsa in Ayurved. Panch kashaya yonivarti having properties like vrana sodhana, kledahara, krimighna, kandughna is selected. The sthanika chikitsa used as the mode of treatment has helped in local cleanliness and prevention of further infections by the microorganism invasion. It had given the natural antibiotic effect without any side effect.

\section{CONCLUSION}

Thus we can conclude that the holistic approach of Ayurvedic system of medicine \& sthanik chikitsa with abhyantara chikitsa plays an important role and gives relief to patient.

\section{REFERENCES}

[1] Text book of Gynecology D.C.Dutta Edited by Hiralal Konar New Central book agency $5^{\text {th }}$ edition.

[2] Charak samhita chaukhamba prakashan chikitasa adhyaya 30

[3] Agnivesha, charaka \& dridhbala.charaka samhita, edited by vaidhya jadhavaji trikamaji acharya. reprint edition, chaukhamba subharati ptrakasana, Varanasi, 2011; chikisa sthanna 30

[4] The Ayurvedic Formulary of India, part 1, Government of India, Ministry of Health and Family Welfare. Department of Indian system of Medicine \& Homeopathy new Delhi:2003

[5] https;//www.medicalnewstoday.com/a rticles/318591.php.

[6] Prof. P V Sharma dravyaguna vigyan, part 2 Delhi; chaukhamba bharti academy, 2013 
[7] Tripathi B, sharanghar samhita purva khanda, Delhi Chaukhambha surbharti 2000,chp 7; pg no:160.

[8] Bhavpraksh madhyamkhanda chikitsa $70 / 47$

[9] Bhavprakash madhyam khanda yonirogadhikara 70/49

[10] https://www.researchgate.net/public ation

[11] Antimicrobial activity of Acorus calamus L. rhizome extract and its total flavonoid and phenolic contents Cite as: AIP Conference Proceedings 2155, 020054 (2019); https://doi.org/10.1063/1.5125558 Published Online: 06 September 2019 Wiwik Susanah Rita, I. Made Dira Swantara and Gusti Ayu Primandani Utami

[12] Samaneh Rahamoz-Haghighi; Antibacterial effect of Acorus calamus extractions against gram positive and negative bacteria Journal homepage:Http://js.kgut.ac.ir

[13] Pratibha Rawal, R.S.Adhikari, K.Danu and A.Tiwari. Antifungal activity of Acorus calamus against Fusarium oxysporum f. sp. lycopersici Kumaun University Nainital, Botany Department,
L.S.M. Govt. P.G. College Pithoragarh, Uttarakhand, India Botany Department, M.B.P.G. College, Haldwani, Uttarakhand, India.Int.J.Curr. Microbiol.App.Sci (2015) 4(1): 710-715 $710 \mathrm{O}$

[14] V. Duraipandiyan, N. A. AlDhabi, C. Balachandran, Antimicrobial, Antioxidant, and Cytotoxic Properties of Vasicine Acetate Synthesized from Vasicine Isolated from Adhatoda vasica $\mathrm{L}$.

[15] Josephin Sheeba, B, Selva mohan;Antimicrobial activity of adhatoda vasica against clinical pathogens;Asian Journals of plant Science and research, 2012, 2(2): 83-88

[16] Hariti M, Rathee PS. Antifungal activity of the unsaponifiable fraction of the fixed oil of Trichosanthes seeds. Asian J Chem 1996;8:180-2.

[17] Hariti M, Rathee PS. Antibacterial activity of the unsaponifiable fraction of the fixed oil of Trichosanthes seeds. Asian J Chem 1995;7:909-11.

[18] Gupta Vikas,Pagoch $s \quad s$ ;Phytopharmacological review of trichosanthes dioicalpatola 
);international ayurvedic medical journal;ISSN;23205091

[19] . Pandey ajay Shankar, srivastava bhavana ,CALLICARPA MACROPHYLLA;Areview of its phytochemistry,pharmacology,folklo re claims and ayurvedic studies;volume3;march2014;91-100

[20] Nitin Kumar, Satyendra Singh, Manvi, and Rajiv Gupta; Trichosanthes dioica Roxb.: An overview; . Pharmacogn Rev. 2012 Jan-Jun; 6(11): 61-67. PMID: 22654406

[21] Virendra Yadav, Soma Jayalakshmi, Rajeev K Singla, Arjun Patra, Salim Khan. Assessment of antiinflammatory and analgesic activities of Callicarpa macrophylla Vahl. roots extracts. Webmedcentral Pharmacology. 2012; 3(5): WMC003366.

[22] Ali Esmail Al-Snafi University of Thi-Qar The pharmacological activities of Cuminumcyminum- $\mathrm{A}$ review Article https://www.researchgate.net/public ation/313742829 July 2016

[23] Burt S. Essential oils: Their antibacterial properties and potential applications in foods: A review. Int
J Food Microbiol. 2004; 94: 22353. [PubMed] [Google Scholar]

[24] Kamaleeswari M, Nalini N. Doseresponse efficacy of caraway on tissue lipid peroxidation and antioxidant profile in rat colon carcinogenesis. $J$ Pharm

Pharmacol. 2006b;58:1121-

30. [PubMed] [Google Scholar]

[25] Thakur S, Bawara B, Dubey A, Nandini D, Chauhan N, Saraf DK. Effect of Carum carvi and Curcuma longa on hormonal and reproductive parameter of female rats. Int $J$ Phytomed. 2009;1:31-8. [Google Scholar]

[26] Sharma $A$ and singh $R$ screening of anti-inflammatory activity of certain indigeneous drug oncarrageenin induced hind paw odema in rats,bull,med ethnobot res $1980 ; 2: 262$

[27] Rao C and Nigam s. Antimicrobial activity of essential oils; Indian $J$ Pharm 1968;30:150

[28] Bhargava $A$ and Chauhan $C$ .Antibecterial activity of essential oils,Indian J Pharm 1968;30:150

[29] Nagasawa $H$, Watanabe $K$, Inatomi $\mathrm{H}$ et al. "Effects of bitter melon (Momordica charantia) or ginger 
rhizome (Zingiber offifinale Rosc.) on spontaneous mammary tumorigenesis in SHN m ice." Am J Clin Med., 2002; 30(2-3): 195-205. [30] Sophon, R., P. Amorn, S. Thianiyavarn, P. Surachai, K. Suntree, 1997. Antibacterial activity of some essential oil. Journal Science Research Chalalongkorn, 22: 13-19.

[31] Fatimah A. Agili Chemical Investigation and Antimicrobial Activity of Essential Oil from Carum roxburghianum ;Australian Journal of Basic and Applied Sciences, 7(2): 723-727, 2013 ISSN 1991-8178. 